\title{
NORMALITY VERSUS COUNTABLE PARACOMPACTNESS IN PERFECT SPACES
}

\author{
BY M. L. WAGE, W. G. FLEISSNER, AND G. M. REED
}

Communicated March 5, 1976

Introduction. The purpose of this announcement is to present, in a unified fashion, solutions to long outstanding questions concerning the relationship between countable paracompactness and normality conditions in perfect spaces. Each section of this paper is the contribution of a single author and is so designated.

It was established in 1951 by Dowker [4] that in perfect spaces (i.e. spaces in which closed sets are $G_{\delta}$-sets), normality implies countable paracompactness. However, the validity of the converse has remained an open question until the present. In particular, the relationships between normality, countable paracompactness, and pseudo-normality in Moore spaces has been of considerable interest ([8], [10], [11], [17], [19], and [20], for example). In this paper, the authors (1) produce an example of a countably paracompact, perfect, nonnormal $T_{3}$-space, (2) produce an example of a pseudo-normal, separable, noncountably paracompact Moore space, and (3) show the consistency and independence of the existence of a countably paracompact, separable, nonnormal Moore space. In addition, several corollaries are given which answer open questions concerning the hereditary and mapping properties of countable paracompactness in perfect spaces.

I. (Wage [17]). The construction given below associates a regular, nonnormal $T_{2}$-space $X^{*}$ to each normal, noncollectionwise normal space $X$.

The MACHINE. Suppose $X$ is a normal $T_{2}$-space and $\left\{H^{\alpha}\right\}_{\alpha<\lambda}$ is a discrete collection of closed sets which cannot be separated by open sets. Let $D=X \backslash H$, where $H=\bigcup\left\{H^{\alpha}: \alpha<\lambda\right\}$. Denote

$$
X^{*}=(X \times\{0,1\}) \cup(D \times\{(\alpha, \beta): \alpha, \beta<\lambda \text { and } \alpha \neq \beta\}) \text {. }
$$

For each $A \subset X$ and $\delta \in\{0,1\} \cup\{(\alpha, \beta): \alpha, \beta<\lambda$ and $\alpha \neq \beta\}$, let $A_{\delta}$ denote $(A \times\{\delta\}) \cap X^{*}$. Now, define a base $B$ for the desired topology on $X^{*}$ as follows

(1) if $x \in X^{*} \backslash\left(H_{0} \cup H_{1}\right)$, let $\{x\} \in B$; and

(2) if $U$ is an open set in $X$ and $\alpha<\lambda$ such that $U \subset\left(H^{\alpha} \cup D\right)$, let

AMS (MOS) subject classifications (1970). Primary 54D15, 54D20, 54G20; Seccondary $02 \mathrm{K05}, 54 \mathrm{C05}, 54 \mathrm{E} 30$. 


$$
\left(\bigcup\left\{U_{(\alpha, \beta)}: \alpha \neq \beta<\lambda\right\} \cup U_{0}\right) \in B
$$

and let

$$
\left(\bigcup\left\{U_{(\beta, \alpha)}: \alpha \neq \beta<\lambda\right\} \cup U_{1}\right) \in B .
$$

Note that $X_{0}$ and $X_{1}$ are two mutually exclusive closed sets in $X^{*}$ which cannot be separated by open sets, hence $X^{*}$ is not normal.

Notation. (CH) will denote the assumption of the continuum hypothesis and $(7 \mathrm{CH})$ will denote the assumption of its negation. The assumption of Martin' Axiom (see [16] and [17]) will be denoted by (M. A.).

THEOREM 1. If $X$ is a normal, noncollectwise normal $T_{2}$-space then $X^{*}$ is a regular, nonnormal $T_{2}$-space and

(1) $X$ perfect $\rightarrow X^{*}$ perfect,

(2) $X$ first countable $\rightarrow X^{*}$ first countable,

(3) $X$ Moore $\rightarrow X^{*}$ Moore,

(4) $X$ collectionwise Hausdorff $\rightarrow X^{*}$ collectionwise Hausdorff,

(5) X countably paracompact $\rightarrow X^{*}$ countably paracompact.

COROLlaRY 1. There exists a perfect $T_{3}$-space which is countable paracompact but not normal.

Proof. Example $\mathrm{H}$ of [1] is a perfectly normal $T_{2}$-space that is not collectionwise normal. Hence, Theorem 1 yields the desired example.

COROLlARY 2. If there exists a normal nonmetrizable Moore space, then there exists a countably paracompact, nonnormal Moore space.

COROLlary 3 (M.A. $+7 \mathrm{CH})$. There exists a countably paracompact Moore space that is not normal.

COROLlARY 4 (M.A. $+7 \mathrm{CH})$. There exists a closed continuous mapping from a countably paracompact Moore space onto a $T_{2}$-space which fails to be countably paracompact.

Corollary 5 (M.A. $+7 \mathrm{CH})$. There exists a countably paracompact Moore space that is not hereditarily countably paracompact.

Corollaries 4 and 5 follow from Theorem 1 and results by Zenor [20].

THEOREM $2(\diamond)$. There exists a nonnormal $T_{3}$-space which is countably compact, perfect, first countable, locally compact, locally countable, zerodimensional, and hereditarily separable.

The construction of the example in Theorem 2 depends heavily on Jensen's $\diamond$ and the technique used by Ostaszewski [9] . 


\section{II. (Fleissner [5]).}

Jones' LEMmA ([7]). If $S$ is a normal $T_{1}$-space, $D$ is a dense subset of $S$, and $Y$ is a closed discrete subset of $S$, then $2^{|Y|} \leqslant 2^{|D|}$.

Lemma F. If $S$ is a countably paracompact $T_{1}$-space, $D$ is a dense subset of $S$, and $Y$ is a closed discrete subset of $S$, then $|Y|<2^{|D|}$.

THEOREM $3(\mathrm{CH})$. Each countably paracompact, separable Moore space $S$ is metrizable.

Proof. By Lemma F, each uncountable subset of $S$ has a limit point. Each Moore space with this property is metrizable [7].

III. (Reed [12]). Using the basic splitting concept of the construction due to Wage in $\S I$, the author of this section was able to construct the examples below which are remarkable for their simplicity.

ExAmple 1 (M.A. $+7 \mathrm{CH}$ ). There exists a countably paracompact separable Moore space which is not normal.

REMARK. Note that Example 1, together with Theorem 3, establishes that the existence of a countably paracompact, separable Moore space is consistent with and independent of the usual axioms of set theory.

EXAmple 2 (M.A. $+7 \mathrm{CH}$ ). There exists a countably paracompact, screenable Moore space which is not normal.

Pseudo-Normality. Proctor [10] defined a space to be pseudo-normal provided each two mutually exclusive closed sets, one of which is countable, can be separated by open sets. Proctor also noted that countably paracompact $T_{3}$ spaces are pseudo-normal and gave an example of a pseudo-normal, separable, nonmetrizable Moore space. Another such example was given by Tall [17] However, to show the noncountable paracompactness of these spaces, one must assume the Continuum Hypothesis. In particular, under M.A. + $7 \mathrm{CH}$, Tall's example is known to be normal. An example of a pseudo-normal, noncountably paracompact, nonseparable Moore space was given in [11]

EXAMPLE 3. There exists a pseudo-normal, separable Moore space which is not countably paracompact.

Remarks on The constructions. A $Q$-set (respectively, $\lambda$-set) is an uncountable subset of the real line in which each subset (respectively, each countable subset) is a relative $G_{\delta}$-set. $Q$-sets exist under M.A. $+7 \mathrm{CH}[16]$ and $\lambda$-sets exist without any extra set-theoretic assumptions beyond the Axiom of Choice [7]. The construction of Example 1 is accomplished by considering a tangent disk space defined on a $Q$-set and splitting the $Q$-set into two disjoint copies which cannot be separated. The construction of Example 2 is done in a similar manner by considering Heath's “ $V$ " space [6] defined on a $Q$-set. Example 3 is constructed by considering a tangent disk space defined on a $\lambda$-set and split- 
ting the $\lambda$-set into countably infinitely many copies. These constructions differ from the construction given in $\S I$ in that the space is not made Hausdorff by splitting points in the upper plane but by assigning disjoint neighborhoods in the same plane to corresponding points on the disjoint copies of the real line.

In [13], it is noted that Example 1 is neither continuously semimetrizable nor submetrizable, while Example 2 (in fact, a space such as Example 2 constructed without any extra set-theoretic assumptions) is continuously semimetrizable but not submetrizable. These examples thus answer questions raised in [2], [3] , and [19] concerning the relationships between continuous semimetrizability, submetrizability, and a zero-set diagonal. Recall that in [14], it was shown that each normal Moore space of cardinality $\leqslant c$ is submetrizable. Also, the construction technique of Example 2 produces the first example of a perfect map from a screenable Moore space onto a nonscreenable Moore space.

\section{REFERENCES}

1. R. H. Bing, Metrization of topological spaces, Canad. J. Math. 3 (1951), 175-186. MR 13, 264.

2. D. K. Burke and D. J. Lutzer, Recent developments in the theory of generalized metric spaces, Proc. Topology Conf. (Memphis State Univ., 1975) (to appear).

3. H. Cook, Cartesian products and continuous semi-metrics, Proc. Conf. on Topology (1967), Arizona State Univ., Tempe, Ariz., 1968, pp. 58-63. MR 38 \#5152.

4. C. H. Dowker, On countably paracompact spaces, Canad. J. Math. 3 (1951), 219224. MR 13, 264.

5. W. G. Fleissner, On discrete subsets of Moore spaces (to appear).

6. R. W. Heath, Screenability, pointwise paracompactness, and metrization of Moore spaces, Canad. J. Math. 16 (1964), 763-770. MR 29 \#4033.

7. F. B. Jones, Concerning normal and completely normal spaces, Bull. Amer. Math. Soc. 43 (1937), 671-677.

8. C. I. Kerr, On countably paracompact spaces, TOPO-General Topology and its Applications (Proc. 2nd. Pittsburgh Internat. Conf., 1972), Lecture Notes in Math., vol. 378, Springer-Verlag, Berlin and New York, 1974, pp. 243-247. MR 49 \#1457.

9. A. J. Ostaszewski, On countably compact, perfectly normal spaces, J. London Math. Soc. (to appear).

10. C. W. Proctor, A separable, pseudonormal, nonmetrizable Moore space, Bull. Acad. Polon. Sci. Sér. Sci. Math. Astronom. Phys. 18 (1970), 179-181. MR 41 \#7628.

11. G. M. Reed, On chain conditions in Moore spaces, General Topology and Appl. 4 (1974), 255-267. MR 49 \#9815.

12. - On normality and countable paracompactness (to appear).

13. - On continuously semi-metrizable and submetrizable spaces (to appear).

14. G. M. Reed and P. L. Zenor, Metrization of Moore spaces and generalized manifolds, Fund. Math. (to appear).

15. F. Slaughter, Submetrizable spaces, Topology Conf. (Virginia Polytech. Inst. and State Univ., 1973), Lecture Notes in Math., vol. 375, Springer-Verlag, Berlin, 1974. MR 49 \#3803.

16. F. Tall, Set-theoretic consistency results and topological theorems concerning the normal Moore space conjecture and related problems, Thesis, Univ. of Wisconsin, 1969.

17. - P-points in $\beta N-N$, normal non-metrizable Moore spaces and other problems of Hausdorff, TOPO-General Topology and Its Applications (Proc. 2nd. Pittsburgh Internat. Conf., 1972), Lecture Notes in Math., vol. 378, Springer-Verlag, Berlin and New York, 1974, pp. 501-512. MR $49 \# 1457$.

18. M. L. Wage, Countable paracompactness, normality, and Moore spaces, Proc. Amer. Math. Soc. 
19. J. N. Younglove, Two conjectures in point set theory, Topology Seminar (Wisconsin, 1965), Ann. of Math. Studies, no. 60, Princeton Univ. Press., Princeton, N.J., 1966, pp. 121-123. MR 36 \#849.

20. P. L. Zenor, On countable paracompactness and normality, Prace Mat. 13 (1969), 24-32. MR $40 \# 1975$.

DEPARTMENT OF MATHEMATICS, UNIVERSITY OF WISCONSIN AT MADISON, MADISON, WISCONSIN 53706

Current address (M. L. Wage): Department of Mathematics, Yale University, New Haven, Connecticut 06520

DEPARTMENT OF MATHEMATICS, MCGILL UNIVERSITY, MONTREAL, QUEBEC H3C 3G1, CANADA

Current address (W. G. Fleissner): Institute for Medicine and Mathematics, Ohio University, Athen, Ohio 45701

INSTITUTE FOR MEDICINE AND MATHEMATICS, OHIO UNIVERSITY, ATHENS, OHIO 45701 